\title{
Crystal-melt interfacial free energy of binary hard spheres from capillary fluctuations
}

\author{
Majeed Amini \\ Department of Physics, University of Kansas, Lawrence, Kansas 66045, USA \\ Brian B. Laird* \\ Department of Chemistry, University of Kansas, Lawrence, Kansas 66045, USA
}

(Received 8 September 2008; published 30 October 2008)

\begin{abstract}
Using molecular-dynamics simulation coupled with an analysis of equilibrium capillary fluctuations in interfacial position, we compute the magnitude and anisotropy of the interfacial free energy $\gamma$ for a binary hard-sphere system with a diameter ratio $\alpha=0.9$. This system, in which the fluid mixture coexists with a randomly substituted face-centered-cubic solid solution, is a useful reference model for alloys. Our results show that $\gamma$ increases with increasing mole fraction of the smaller sized particle when temperature is held constant. However, after rescaling the results to fixed pressure and varying temperature, we find that $\gamma$ decreases with increased alloying by the smaller particle (corresponding to lower temperatures). Thus, $\gamma$ is seen to decrease with increasing concentration of the lower melting point solute, consistent with earlier simulations on $\mathrm{Ni} / \mathrm{Cu}$ and Lennard-Jones mixtures. The anisotropy in $\gamma$ is such that the inequality $\gamma_{100}>\gamma_{110}>\gamma_{111}$ holds for all concentrations studied. Using the classification scheme of Haxhimali et al., [Nat. Mater. 5, 660 (2006)] we find that the anisotropy in $\gamma$ is consistent with a predicted $\langle 100\rangle$ primary dendrite growth direction.
\end{abstract}

DOI: 10.1103/PhysRevB.78.144112

PACS number(s): 68.35.Md, 05.70.Np, 68.08.De

\section{INTRODUCTION}

The crystal-melt interfacial free energy, $\gamma$, is a primary thermodynamic parameter governing the kinetics and morphology of crystal growth from the melt, ${ }^{1}$ and is defined as the reversible work required to form a unit area of interface between a crystal and a coexisting fluid. ${ }^{2}$ Accurate values of $\gamma$ are necessary for a full understanding of a number of technologically important interfacial phenomena in chemistry and materials science. For example, the orientation dependence of $\gamma$, although generally small (1-4\% for most metals), has a significant effect on the shape and kinetics of growing dendrites. ${ }^{3-7}$

Experimentally, the majority of estimates for $\gamma$ are obtained indirectly from measurements of nucleation kinetics, interpreted using classical nucleation theory. ${ }^{2,8,9}$ Such analyses typically underestimate $\gamma$ by about 10-20\%. Using this method, Turnbull ${ }^{9}$ reported the values of $\gamma$ for variety of materials, mostly metallic elements. For pure materials, the values of $\gamma$ determined by Turnbull exhibit a strong empirical correlation with the latent heat of fusion $\left(\Delta H_{\text {fus }}\right)$, given by the relation $\gamma \approx C_{T} \Delta H_{\text {fus }} \rho^{2 / 3}$, where $\rho$ is the number density of the crystal, and $C_{T}$ is the so-called Turnbull coefficient, which was reported by Turnbull to be approximately 0.45 for metals and 0.32 for many nonmetals and semimetals. Estimates of $\gamma$ from nucleation rates are orientational averages and, thus, cannot be used to determine the anisotropy in this quantity. More accurate direct experimental measurements involving contact angle studies are quite difficult to perform and are, subsequently, relatively few in number. ${ }^{10}$ In principle, contact angle measurements can be used to determine the anisotropy in $\gamma$, but, in practice, with a few exceptions, ${ }^{11,12}$ they are not sufficiently precise for this purpose. Recently, Napolitano et al. developed a quantitative technique for measuring equilibrium crystal shapes from which, through the use of a Wulff construction, ${ }^{8}$ the anisotropy (but not the magnitude) of $\gamma$ can be determined accu- rately. They have applied their methods to $\mathrm{Al}-\mathrm{Si},{ }^{13} \mathrm{Al}-\mathrm{Cu},{ }^{14}$ and Al-Sn (Ref. 15) binary mixtures. In the later study, the full three-dimensional Wulff construction was obtained, allowing them to thoroughly map out the anisotropy of the Al-Sn system.

The lack of accurate experimental measurements has motivated the development of a variety of molecular simulation methods to determine $\gamma$ computationally. This effort has focused largely on two complimentary classes of simulation techniques: cleaving methods, based on thermodynamic integration, ${ }^{16-18}$ and methods based on an analysis of capillary fluctuations in interface position. ${ }^{19}$ Applications of these methods have focused both on the determination of $\gamma$ for realistic models of materials (e.g., metals ${ }^{19-21}$ or organic materials ${ }^{22}$ ) and for simple models, such as hard spheres, ${ }^{17}$ soft spheres, ${ }^{18}$ and Lennard-Jones particles. ${ }^{23-25}$

Most simulation studies, however, have focused primarily on pure materials, and, to date, only a small number of studies have examined alloys. ${ }^{7,26}$ In this work, we examine the magnitude and anisotropy of a binary hard-sphere mixture, composed of two components with differing particle diameters in a ratio of 0.9 . There are two main reasons for our choice of system: first, hard spheres are an important reference system for simple fluids ${ }^{27}$ and have shown to be an accurate model for the interfacial thermodynamics of closepacked crystal-forming pure materials, ${ }^{28}$ Second, Kranendonk and Frenkel ${ }^{29}$ have determined accurate pressurecomposition phase diagrams for binary hard spheres with diameter ratios of $0.85,0.90$, and 0.95 . This is crucial because any study of interfacial thermodynamics must start with an accurate phase diagram to correctly prepare a system in solid-liquid coexistence.

In 2002, Asta et al. ${ }^{26}$ used a capillary fluctuation method (CFM) to determine the interfacial free energy of a $\mathrm{Ni} / \mathrm{Cu}$ alloy, at zero pressure, with equilibrium solidus and liquidus copper mole fractions of 0.050 and 0.104 , respectively. Their simulations were performed using an embedded atom model 
(EAM) potential for $\mathrm{Ni} / \mathrm{Cu}$ developed by Foiles. ${ }^{30}$ Their model alloy system was found to have an orientationally averaged $\gamma$ of $287 \pm 8 \mathrm{~mJ} \mathrm{~m}^{-2}$, which is about $7.4 \%$ lower than that calculated for pure Ni. Thus, in this work, the value of $\gamma$ is predicted to decrease upon the addition of a lower melting point solute $(\mathrm{Cu})$. In addition, the anisotropy in $\gamma$ for this alloy system was found to be decreased slightly from that of pure $\mathrm{Ni}$.

More recently, in 2007, Becker et al. ${ }^{7}$ examined, via simulation, the magnitude and anisotropy of $\gamma$ for a binary Lennard-Jones mixture. The interaction potential for this system is

$$
V\left(r_{i j}\right)=4 \epsilon_{i j}\left[\left(\frac{\sigma_{i j}}{r_{i j}}\right)^{12}-\left(\frac{\sigma_{i j}}{r_{i j}}\right)^{6}\right],
$$

where $i$ and $j$ index particle types (1 or 2) and $\sigma_{i j}$ and $\epsilon_{i j}$ determine the size and energy scales for the interaction. Becker et al. examined a system with no size anisotropy (that is, $\left.\sigma_{11}=\sigma_{22}\right)$, but with a well-depth ratio $\epsilon_{11} / \epsilon_{22}$ equal to 0.75. Standard Lorentz-Berthelot mixing rules were used for the mixed interactions. Like the $\mathrm{Ni} / \mathrm{Cu}$ system studied by Asta et al. ${ }^{26}$ this LJ mixture exhibits nearly ideal solution behavior in the solid and liquid. Through an analysis of capillary fluctuations, Becker et al., determined that the orientationally averaged value of $\gamma, \gamma_{0}$, varied smoothly from $0.355(8)$ to $0.267(8) \epsilon_{22} / \sigma_{22}^{2}$ as the mole fraction of component $2\left(x_{2}\right)$ was decreased from 1 to 0 . The values for the pure systems $\left(x_{2}=1\right.$ or 0$)$ are in agreement with previous results from both CFM (Ref. 25) and cleaving. ${ }^{16,24}$ These results are consistent with those by Asta et al. ${ }^{26}$ for $\mathrm{Ni} / \mathrm{Cu}$ in that the interfacial free energy is decreased upon the addition of a lower melting point solute. In addition, although the changes in $\gamma$ anisotropy were seen to be small, they were predicted to be sufficiently large to affect qualitative changes in the dendrite morphology. These results complement the current study because, while Becker et al. examined the effect on $\gamma$ on the alloying of two-particle types of identical size but differing energy scales, the current study is focused on the effect of alloying of two-particle types that differ in size, but have equal energy scales.

\section{SYSTEM}

The interaction energy for the binary hard-sphere system is defined as

$$
V\left(r_{i j}\right)=\left\{\begin{array}{ll}
\infty, & r_{i j}<\sigma_{i j} \\
0, & r_{i j} \geq \sigma_{i j}
\end{array},\right.
$$

where $i$ and $j$ index particle type ( 1 or 2 ). We assume that the hard spheres are additive, so that $\sigma_{12}=\sigma_{21}$ is the arithmetic mean of $\sigma_{11}$ and $\sigma_{22}$. This system is completely specified by the total particle density, $\rho$, the mole fraction of one of the particles (here $x_{2}$ is used), and the diameter ratio $\alpha$ $=\sigma_{11} / \sigma_{22}$, which, without loss of generality, is assumed to be less than unity (that is, the type 1 particle is assumed to be the smaller of the two-particle types). For convenience, we define the reduced number density $(\rho=N / V)$ using the larger particle diameter $\left(\sigma_{22}\right)$ to define the length scale

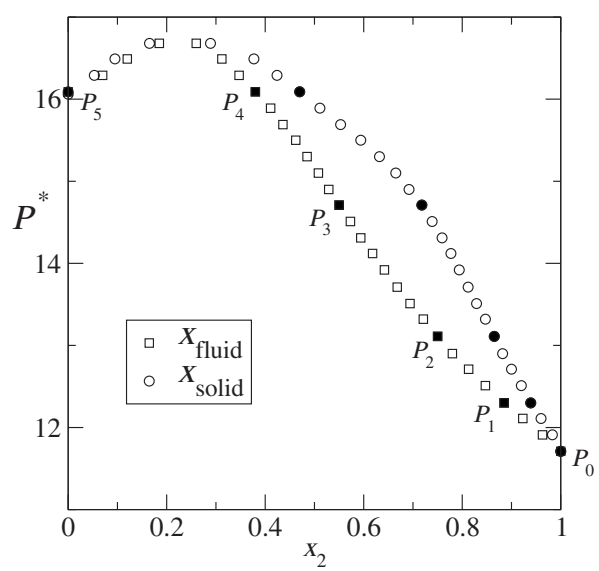

FIG. 1. Phase diagram of a binary hard-sphere system with $\alpha$ $=0.9$. The data are taken from simulation by Kranendonk and Frenkel (Ref. 29). The melt and crystal are represented by squares and circles, respectively. The solid squares and circles correspond to the coexistence states used in the present work.

$$
\rho^{*} \equiv \rho \sigma_{22}^{3}=\rho_{1}^{*}+\rho_{2}^{*},
$$

where $\rho_{1}^{*}$ and $\rho_{2}^{*}$ are the similarly defined reduced number densities for the small and large particles, respectively. Another useful measure is the packing fraction, which is defined as the fraction of the total volume of the system occupied by the particles

$$
\eta=\eta_{1}+\eta_{2}
$$

where the individual packing fractions $\eta_{1}$ and $\eta_{2}$ are $\pi \alpha^{3} \rho_{1}^{*} / 6$ and $\pi \rho_{2}^{*} / 6$, respectively. Using $\sigma_{22}$ as the length scale and noting that the only energy scale in the system is $k_{B} T$, where $k_{B}$ is Boltzmann's constant and $T$ is the temperature, we can define the reduced pressure and interfacial free energy as

$$
\begin{gathered}
P^{*}=\frac{P \sigma_{22}^{3}}{k_{B} T}, \\
\gamma^{*}=\frac{\gamma \sigma_{22}^{2}}{k_{B} T} .
\end{gathered}
$$

For this initial study, we choose a system with a diameter ratio of $\alpha=0.9$. The reasons for this choice are twofold. First, the phase diagram for this diameter ratio was determined previously by Kranendonk and Frenkel. ${ }^{29,31}$ Second, the structure and dynamics of the system have been well characterized. ${ }^{32}$ The pressure or composition phase diagram is shown in Fig. 1. At this diameter ratio (0.9), solid-fluid coexistence in the binary hard sphere exhibits significant nonideal behavior, as seen by the presence of an azeotrope in Fig. 1. The filled symbols in Fig. 1 represent the phase points for which we have obtained estimates of the interfacial free energy. For these points the phase-coexistence data used in this study are summarized in Table I. We have not included the azeotrope in this initial study. Over the entire composition range, the solid phase for the mixtures is a randomly substituted face-centered cubic crystal. The precise pressures 
TABLE I. The reduced pressure $\left(P^{*}\right)$, fluid and crystal packing fraction $\left(\eta_{f}, \eta_{c}\right)$, fluid and crystal mole-fraction $\left(x_{f}, x_{f}\right)$, from simulation, are shown. The $P_{i}$ refers to the points on Fig. 1 .

\begin{tabular}{lcllll}
\hline \hline System & $P^{*}\left(k_{B} T / \sigma^{2}\right)$ & \multicolumn{1}{c}{$\eta_{f}$} & \multicolumn{1}{c}{$\eta_{c}$} & \multicolumn{1}{c}{$x_{f}$} & \multicolumn{1}{c}{$x_{c}$} \\
\hline$P_{0}$ & 11.57 & 0.491 & 0.543 & 1.0 & 1.0 \\
$P_{1}$ & 12.23 & 0.4963 & 0.548 & 0.89 & 0.947 \\
$P_{2}$ & 13.13 & 0.4998 & 0.5498 & 0.75 & 0.865 \\
$P_{3}$ & 14.7 & 0.502 & 0.552 & 0.54 & 0.71 \\
$P_{4}$ & 16.19 & 0.510 & 0.549 & 0.38 & 0.47 \\
$P_{5}$ & 15.87 & 0.491 & 0.543 & 1.0 & 1.0 \\
\hline \hline
\end{tabular}

and compositions in Table I differ slightly from those of Kranendonk and Frenkel ${ }^{31}$ —we have optimized the phase coexistence parameters to ensure a stress-free crystal phase and a stable interface, see Ref. 33 for a discussion of the procedure used. It should be noted that the phase points $P_{5}$ $\left(x_{2}=0\right)$ and $P_{0} \quad\left(x_{2}=1\right)$ are representations of a singlecomponent hard-sphere system and are thus identical when scaled by their respective length scales.

\section{METHOD}

At present, most calculations of the crystal-melt interfacial free energy via computer simulation involve one of two qualitatively different approaches: thermodynamic integration or capillary fluctuation analysis. For a recent review, see Ref. 34. Thermodynamic integration was first applied in 1986 to the solid-liquid interface by Broughton and Gilmer, ${ }^{16}$ who utilized specially constructed cleaving potentials to calculate $\gamma$ for the Lennard-Jones system. This method was modified in 2000 by Davidchack and Laird, ${ }^{17}$ replacing the complex cleaving potentials of Broughton and Gilmer with cleaving walls made up of particles that are identical or similar to the atoms of the system of interest. This "cleaving wall" method has been used to determine $\gamma$ for a number of systems including hard spheres, ${ }^{17,18}$ Lennard-Jones particles, ${ }^{24,35}$ repulsive soft spheres, ${ }^{18}$ and, most recently, water. ${ }^{36}$ Unfortunately for the present work, the cleaving method is not well suited to mixtures (except, perhaps, at an azeotrope) because of the difficulty in constructing cleaving walls that properly partition the interface with respect to the differing composition of liquid and crystal.

The second approach is the capillary fluctuation method in which the interfacial free energy is determined by examining the power spectrum of fluctuations in interfacial position. ${ }^{19}$ This is the approach that we use in this work, as it is well suited to mixtures. In most cases, the simulation box is a thin slab, quite long in the direction normal to the interface, but only a few lattice spacings in depth. Thus, the interfacial position is a quasi-one-dimensional (quasi-1D) curve, $h(x)$, where $x$ measures the distance along the width of the simulation box, that is, in the direction perpendicular to both the short direction and the interface normal. For a microscopically rough interface, statistical mechanics can be used to obtain ${ }^{37}$ a relationship between $h(x)$ and the interfacial stiffness, $\widetilde{\gamma}$

$$
\left\langle|h(q)|^{2}\right\rangle=\frac{k_{B} T}{b W \tilde{\gamma} q^{2}},
$$

where $h(q)$ is the 1D Fourier transform of $h(x), W$ and $b$ denote the width and depth of the simulation box, respectively, $k_{B}$ is the Boltzmann constant, and $T$ is the melting temperature of the bulk. The interfacial stiffness, $\tilde{\gamma}$, is defined as ${ }^{38}$

$$
\tilde{\gamma}=\gamma(\theta)+\frac{d^{2} \gamma}{d \theta^{2}}
$$

where $\theta$ is the angle between the instantaneous and average interface normal. The first term in the definition of $\tilde{\gamma}, \gamma$ determines the energy required to change the length of the interface boundary, whereas the second term $\frac{d^{2} \gamma}{d \theta^{2}}$ determines the energy required for changes in local interfacial orientation. One advantage to measuring $\tilde{\gamma}$ rather than $\gamma$ itself is that $\tilde{\gamma}$ is significantly more anisotropic than $\gamma$ itself, allowing a more precise resolution of interface anisotropy. Once $\tilde{\gamma}$ has been determined for several interfacial orientations, the corresponding values of $\gamma$ can be obtained by first parametrizing the orientation dependence of $\gamma$ and then using the data for $\tilde{\gamma}$ to determine the optimal parameter fit, from which the value of $\gamma$ at a variety of orientations can be determined. For our parametrization we use the cubic-harmonic expansion due to Fehlner and Vosko, ${ }^{39}$ which has been used successfully in similar calculations on a variety of systems ${ }^{19,22}$

$$
\gamma(\hat{\mathbf{n}}) / \gamma_{0}=1+\epsilon_{1}\left(\sum_{i=1}^{3} n_{i}^{4}-\frac{3}{5}\right)+\epsilon_{2}\left(3 \sum_{i=1}^{3} n_{i}^{4}+66 \prod_{i=1}^{3} n_{i}^{2}-\frac{17}{7}\right)
$$

where $\left(n_{1}, n_{2}, n_{3}\right)$ are the Cartesian components of the interface normal $\hat{\mathbf{n}}, \gamma_{0}$ is the value of $\gamma$ averaged over orientations, and $\epsilon_{1}$ and $\epsilon_{2}$ are parameters describing the anisotropy in $\gamma$.

To implement the CFM it is necessary to determine the interface position, $h(x)$, which requires a method for defining individual atoms as "solid" or "liquid." In this work, we use a local order parameter proposed by Morris, ${ }^{20}$ as modified in Ref. 40. For each configuration, the two interfacial position functions, $h(x, t)$ are determined. For each $h(x, t)$, the Fourier amplitude, $\tilde{h}(q, t)$, was determined using FFT.

The CFM has been used to determine $\gamma$ for a number of materials, such as $\mathrm{Ni},{ }^{19} \mathrm{Al},{ }^{20} \mathrm{Ni}-\mathrm{Cu},{ }^{26} \mathrm{Ag}$ and $\mathrm{Au},{ }^{41}$ hard spheres, ${ }^{40}$ and Lennard-Jones particles. ${ }^{25}$ For the hard-sphere and Lennard-Jones systems, the CFM has been found to give results that agree within simulation error with cleaving wall calculations on the same systems. ${ }^{25,40}$ In general, CFM calculations require larger system sizes (40 000-100 000 particles) than the cleaving wall method. Although the cleaving wall method is generally more precise in its determination of the magnitude of $\gamma$ than the CFM, the CFM can determine the anisotropy in $\gamma$ to a higher precision than is possible in cleaving. This is due to the fact that the CFM determines directly the interfacial stiffness, $\tilde{\gamma}$, which is much more anisotropic than $\gamma$, itself. 
TABLE II. The results for the interfacial stiffness, $\tilde{\gamma}$, for the five pressures listed in Table I for each of the four orientations examined.

\begin{tabular}{cllll}
\hline \hline Pressure & \multicolumn{5}{c}{ Stiffness $\tilde{\gamma}\left(k T / \sigma_{2}^{2}\right)$} \\
$P\left(k T / \sigma_{2}^{3}\right)$ & $(100)[010]$ & $(110)[1 \overline{1} 0]$ & $(110)[001]$ & $(111)[1 \overline{1} 0]$ \\
\hline 11.57 & $0.44(5)$ & $0.458(12)$ & $0.72(3)$ & $0.76(3)$ \\
12.23 & $0.450(7)$ & $0.447(11)$ & $0.72(4)$ & $0.849(13)$ \\
13.13 & $0.468(16)$ & $0.494(12)$ & $0.79(3)$ & $0.806(17)$ \\
14.70 & $0.49(3)$ & $0.467(17)$ & $0.84(6)$ & $0.87(3)$ \\
16.19 & $0.415(12)$ & $0.43(4)$ & $0.928(7)$ & $0.926(9)$ \\
\hline \hline
\end{tabular}

\section{RESULTS AND DISCUSSION}

For our current molecular-dynamics (MD) simulations of the $\alpha=0.9$ binary hard-sphere system, we created samples of coexisting crystal and melt for the pressures and compositions listed in Table I (with the exception of $P_{5}$, which is identical to $P_{0}$ after scaling). For each pressure, simulation cells for four different interfacial orientations were constructed: (100)[010], (110)[1̄0], (110)[001], and (111)[1ํㅣ, where the notation $(i j k)$ denotes the interface normal $(z)$ and $[h k l]$ denotes the short direction $(y)$ of the box. In all simulations, the short direction of the simulation box $(b)$ is about four to five fcc unit-cell spacings-small enough that the interface position is quasi-one-dimensional. The width $(W)$ of the simulation box is in the range 10-15 times $b$ to ensure that the range of the wave number $q$ is sufficient to determine $\tilde{\gamma}$ from the power spectrum [Eq. (4)]. The length of the simulation box normal to the interfacial plane is chosen to be about 20 times the short direction, which was determined to be sufficient to ensure that the bulk behavior is obtained in the interior of the fluid and crystal phases. The MD simulations were performed using the hardsphere algorithm of Rapaport. ${ }^{42}$ To ensure the construction of stress-free equilibrium crystal-melt interfaces, we use the protocol outlined in Ref. 33. For all interfacial orientations, the number of particles used is approximately 40000 with simulation box dimensions averaging $5.5 \sigma \times 63 \sigma \times 105 \sigma$, these numbers vary slightly from orientation to orientation, depending upon the system geometry.

After equilibration, each sample was simulated for 16500 $\tau$ to collect averages, where $\tau=\sqrt{m \sigma_{2}^{2} / k_{B} T}$ is a natural time unit for binary hard spheres. The mass, $m$, is assumed to be
TABLE III. Expressions for the interfacial free energy and stiffness from Eq. (5), The notation $(i j k)$ denotes the interface, and $[h k l]$ denotes the short direction (see Ref. 25).

\begin{tabular}{lcc}
\hline \hline Interface & $\gamma / \gamma_{0}$ & $\tilde{\gamma} / \gamma_{0}$ \\
\hline$(100)[010]$ & $1+\frac{2}{5} \epsilon_{1}+\frac{4}{7} \epsilon_{2}$ & $1-\frac{18}{5} \epsilon_{1}-\frac{80}{7} \epsilon_{2}$ \\
$(110)[1 \overline{1} 0]$ & $1-\frac{1}{10} \epsilon_{1}-\frac{13}{14} \epsilon_{2}$ & $1-\frac{21}{10} \epsilon_{1}+\frac{365}{14} \epsilon_{2}$ \\
$(110)[001]$ & $1-\frac{1}{10} \epsilon_{1}-\frac{13}{14} \epsilon_{2}$ & $1+\frac{39}{10} \epsilon_{1}+\frac{155}{14} \epsilon_{2}$ \\
$(111)[1 \overline{1} 0]$ & $1-\frac{14}{15} \epsilon_{1}+\frac{64}{63} \epsilon_{2}$ & $1+\frac{12}{5} \epsilon_{1}-\frac{1280}{63} \epsilon_{2}$ \\
\hline \hline
\end{tabular}

identical for all particles, independent of particle type. Snapshot configurations were sampled every $3.3 \tau$, giving a total of 5000 configurations for the data analysis. For each pressure and orientation, the interfacial stiffnesses were calculated using Eq. (4) from a log-log plot of $\langle\tilde{h}(q)\rangle$ versus $q$. These are tabulated in Table II.

Once the interfacial stiffnesses for a given pressure are known for several orientations, the magnitude and anisotropy of the interfacial free energy is obtained by fitting the stiffness data to the three parameters of the Fehlner and Vosko cubic-harmonic expansion [Eq. (6)]: $\gamma_{0}, \epsilon_{1}$, and $\epsilon_{2}$. The specific expressions ${ }^{25}$ for both $\gamma$ and $\tilde{\gamma}$ in terms of the FehlnerVosko parameters are shown in Table III for each of the orientations studied. The fitting is performed using leastsquares linear regression. The results are summarized in Table IV. For the single-component system $\left(P \sigma_{2}^{3} / k T\right.$ $=14.70$ ), our results are in agreement (within the $2 \sigma$ error bars) with the results of Ref. 40 .

Figure 2 shows $\gamma_{0}, \epsilon_{1}$, and $\epsilon_{2}$ as a function of coexistence pressure (at fixed temperature) for the $\alpha=0.9$ binary hardsphere system. This plot shows that, at constant temperature, the orientationally averaged interfacial free energy increases with increasing pressure, and therefore with increasing mole fraction of the smaller diameter particle $\left(x_{1}\right)$. The anisotropy parameter $\epsilon_{1}$ is constant at low pressures but increases as the system approaches the azeotrope in Fig. 1. The anisotropy parameter $\epsilon_{2}$ is, to a good approximation, independent of alloying in this system. For comparison, the anisotropy measures $\left(\gamma_{100}-\gamma_{111}\right) / 2 \gamma_{0}$ and $\left(\gamma_{110}-\gamma_{111}\right) / 2 \gamma_{0}$ are also shown in Table IV. From this data, the following inequality is shown to hold or all pressures (and compositions) studied:

$$
\gamma_{100}>\gamma_{110}>\gamma_{111}
$$

TABLE IV. The calculated orientationally averaged interfacial free energy $\left(\gamma_{0}\right)$, Fehlner and Vosko anisotropy parameters $\left(\epsilon_{1}\right.$ and $\left.\epsilon_{2}\right)$, and anisotropy differences for the binary hard-sphere system for a variety of pressures (and compositions).

\begin{tabular}{rlllcc}
\hline \hline$P\left(k T / \sigma_{2}^{3}\right)$ & $\gamma_{0}\left(k T / \sigma_{2}^{2}\right)$ & \multicolumn{1}{c}{$\epsilon_{1}$} & \multicolumn{1}{c}{$\epsilon_{2}$} & $\left(\gamma_{100}-\gamma_{111}\right) / 2 \gamma_{0}$ & $\left(\gamma_{110}-\gamma_{111}\right) / 2 \gamma_{0}$ \\
\hline 11.57 & $0.591(17)$ & $0.075(12)$ & $-0.0036(13)$ & $0.051(8)$ & $0.038(5)$ \\
12.23 & $0.617(9)$ & $0.078(6)$ & $-0.0062(8)$ & $0.053(4)$ & $0.043(3)$ \\
13.13 & $0.633(9)$ & $0.077(5)$ & $-0.0030(7)$ & $0.052(3)$ & $0.037(2)$ \\
14.70 & $0.663(18)$ & $0.085(10)$ & $-0.0048(13)$ & $0.058(7)$ & $0.044(5)$ \\
16.19 & $0.667(11)$ & $0.117(5)$ & $-0.0047(12)$ & $0.079(3)$ & $0.057(3)$ \\
\hline
\end{tabular}




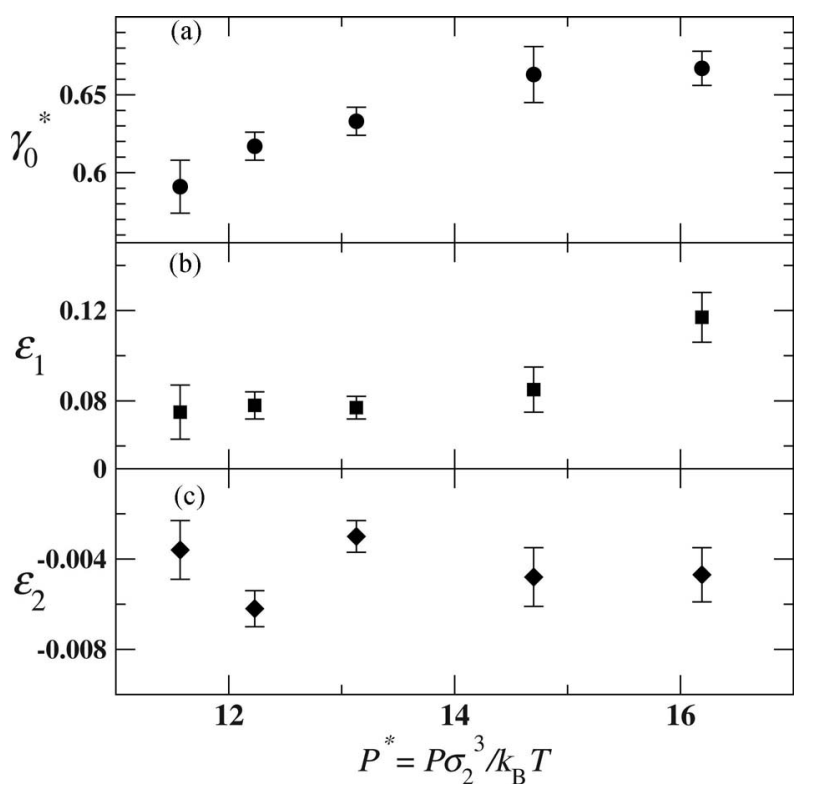

FIG. 2. Results for the interfacial free energy of a $\alpha=0.9$ binary hard-sphere system as a function of coexistence pressure: (a) orientationally averaged reduced interfacial free energy, $\gamma_{0}^{*}=\gamma_{0} \sigma_{2}^{2} / k_{B} T$, (b) anisotropy parameter $\epsilon_{1}$, and (c) anisotropy parameter $\epsilon_{2}$.

Recently, Haxhimali et al. ${ }^{6}$ used phase-field simulations to correlate dendritic growth morphology with the anisotropy of the interfacial free energy. Based on this analysis, our calculated values of $\epsilon_{1}$ and $\epsilon_{2}$ for the binary hard-sphere system $(\alpha=0.9)$ are consistent with the formation of dendrites with a $\langle 100\rangle$ primary growth direction. In the binary Lennard-Jones simulations of Becker et al., ${ }^{7}$ the calculated anisotropies in $\gamma$ indicated a similar $\langle 100\rangle$ primary dendrite growth direction, although the LJ system approaches the $\langle 100\rangle /$ hyperbranched dendrite boundary as the system approaches a 50:50 mixture. In the LJ simulations, which have energy anisotropy but no size anisotropy, the anisotropy parameter $\epsilon_{1}$ is roughly independent of concentration, whereas, $\epsilon_{2}$ shows considerable variation. Thus, it can be speculated that size asymmetry affects $\epsilon_{1}$ primarily, but energy asymmetry has more of an effect on the value of $\epsilon_{2}$.

In Table IV and Fig. 2, the data for $\gamma_{0}$ for binary hardsphere systems are shown as a function of pressure at fixed temperature, $T$. This makes comparison difficult with the $\mathrm{Ni} / \mathrm{Cu}$ simulations of Asta et al.,${ }^{26}$ or the binary LJ simulations of Becker et al., ${ }^{7}$ because in those studies the concentration changes are performed at fixed pressure by varying the coexistence temperature. Due to the unique scaling behavior of hard spheres (that is, both the pressure and interfacial free-energy scale linearly with temperature along the coexistence curve) it is possible to scale the hard-sphere data to vary coexistence temperature at constant pressure. If we fix the pressure to equal the single-component pressure, $P_{0}$, then we can scale the interfacial free energy as follows:

$$
\gamma_{P, 0}=\gamma_{0} \times \frac{P_{0}}{P},
$$

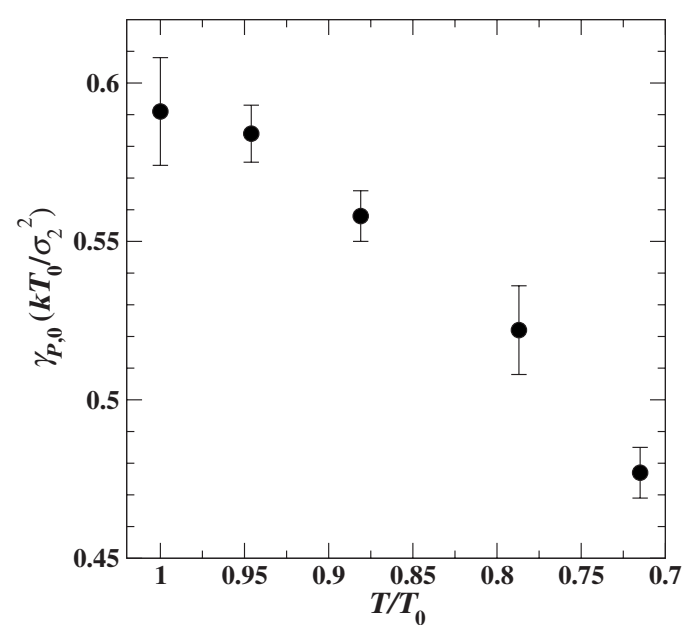

FIG. 3. The orientationally averaged interfacial free energy versus temperature (relative to the single-component temperature) for the binary hard-sphere system in which the concentration of solute is increased at fixed pressure. The solute concentration increases from left to right in the plot.

where $\gamma_{0}$ is the orientationally averaged interfacial free energy measured at a constant $T$ (here equal to $T_{0}$, the coexistence temperature of the pure system) and $\gamma_{P, 0}$ is the interfacial free energy measured at constant pressure (here equal to $\left.P_{0}\right)$ with a temperature $T=T_{0} \times\left(P_{0} / P\right)$. The units of $\gamma_{P, 0}$ are $k T_{0} / \sigma_{2}^{2}$. Note that, the values of $\epsilon_{1}$ and $\epsilon_{2}$, being dimensionless, are unaffected by the scaling. Figure 3 shows $\gamma_{P, 0}$ plotted as a function of temperature relative to the singlecomponent temperature, $T / T_{0}$. As expected, at fixed pressure the melting temperature is lowered as the solute concentration is increased. In this plot, we see that the interfacial free energy decreases as the concentration of lower melting point material (type 1) is added at fixed pressure. This is consistent with both the $\mathrm{Ni} / \mathrm{Cu}$ (Ref. 26) and binary LJ results. ${ }^{7}$

\section{SUMMARY}

Through an analysis of capillary fluctuations in interfacial position, we have determined the diordered-fcc-crystal/melt interfacial free energy, $\gamma$, for a binary hard-sphere mixture with a diameter ratio, $\alpha=\sigma_{11} / \sigma_{22}=0.9$. The value of $\gamma$ was determined at several points along the pressure-composition phase diagram, which has been previously determined by Kranendonk and Frenkel. ${ }^{29}$ For this system, the magnitude of $\gamma$ is shown to increase when mole fraction of the smaller component $\left(x_{1}\right)$ is increased at constant temperature. For comparison to two other such simulations on $\mathrm{Ni} / \mathrm{Cu}$ (Ref. 26) and Lennard-Jones ${ }^{7}$ binary mixtures, both of which involve changing composition at constant pressure, we have used the scaling relations for hard-sphere systems to transform our data to constant pressure. After rescaling, we find that the magnitude of $\gamma$ decreases with increasing $x_{1}$ at constant pressure, which is consistent with both the $\mathrm{Ni} / \mathrm{Cu}$ and $\mathrm{LJ}$ simulations in that the interfacial free energy decreases with addition of a lower melting point solute at constant pressure. 
Our results for the anisotropy in $\gamma$ show that the inequality $\gamma_{100}>\gamma_{110}>\gamma_{111}$ holds for all pressures (and compositions) studied. This is consistent with previous results on $\mathrm{Ni} / \mathrm{Cu}$ (Ref. 26) and Lennard-Jones. ${ }^{7}$ Our results also show that the anisotropy in $\gamma$ for the $\alpha=0.9$ binary mixtures increases with increasing $x_{1}$, approaching the azeotrope. Using the recent work of Haxhimali et al. ${ }^{6}$ which correlated the anisotropy in $\gamma$ with expected dendrite growth morphology, we find that the calculated anisotropy of this binary hardsphere system is consistent with a primary dendrite growth direction of $\langle 100\rangle$.

\section{ACKNOWLEDGMENTS}

The authors wish to thank Ruslan Davidchack and Marc Odunlami for kindly providing us with their MD code for hard spheres and Mark Asta for helpful discussions. In addition, we gratefully acknowledge partial support for this work from the National Science Foundation under Grant No. CHE0316127. Finally, this work has benefited greatly from the participation of one of the authors (B.B.L.) in meetings sponsored by the Computational Materials Science Network, a Department of Energy funded collaboration.

\footnotetext{
*blaird@ku.edu

${ }^{1}$ J. J. Hoyt, M. Asta, T. Haxhimali, A. Karma, R. E. Napolitano, R. Trivedi, B. B. Laird, and J. R. Morris, MRS Bull. 29, 935 (2004).

${ }^{2}$ W. A. Tiller, The Science of Crystallization: Microscopic Interfacial Phenomena (Cambridge University Press, New York, 1991).

${ }^{3}$ A. Karma and W. J. Rappel, Phys. Rev. E 57, 4323 (1998).

${ }^{4}$ A. Karma, Phys. Rev. Lett. 87, 115701 (2001).

${ }^{5}$ W. J. Boettinger, S. R. Coriell, A. L. Greer, A. Karma, W. Kurz, M. Rappaz, and R. Trivedi, Acta Mater. 48, 43 (2000).

${ }^{6}$ T. Haxhimali, A. Karma, F. Gonzales, and M. Rappaz, Nature Mater. 5, 660 (2006).

${ }^{7}$ C. A. Becker, D. Olmsted, M. Asta, J. J. Hoyt, and S. M. Foiles, Phys. Rev. Lett. 98, 125701 (2007).

${ }^{8}$ D. P. Woodruff, The Solid-Liquid Interface (Cambridge University Press, London, 1973).

${ }^{9}$ D. Turnbull, J. Appl. Phys. 21, 1022 (1950).

${ }^{10}$ J. M. Howe, Interfaces in Materials (Wiley, New York, 1997).

${ }^{11}$ M. E. Glicksman and N. B. Singh, J. Cryst. Growth 98, 277 (1989).

${ }^{12}$ M. Muschol, D. Liu, and H. Z. Cummins, Phys. Rev. A 46, 1038 (1992).

${ }^{13}$ S. Liu, R. E. Napolitano, and R. Triveti, Acta Mater. 49, 4271 (2001).

${ }^{14}$ R. E. Napolitano, S. Liu, and R. Triveti, Interface Sci. 10, 217 (2002).

${ }^{15}$ R. E. Napolitano and S. Liu, Phys. Rev. B 70, 214103 (2004).

${ }^{16}$ J. Q. Broughton and G. H. Gilmer, J. Chem. Phys. 84, 5741 (1986).

${ }^{17}$ R. L. Davidchack and B. B. Laird, Phys. Rev. Lett. 85, 4751 (2000).

${ }^{18}$ R. L. Davidchack and B. B. Laird, Phys. Rev. Lett. 94, 086102 (2005).

${ }^{19}$ J. J. Hoyt, M. Asta, and A. Karma, Phys. Rev. Lett. 86, 5530 (2001).
}

${ }^{20}$ J. R. Morris, Phys. Rev. B 66, 144104 (2002)

${ }^{21}$ D. Y. Sun, M. Asta, J. J. Hoyt, M. I. Mendelev, and D. J. Srolovitz, Phys. Rev. B 69, 020102(R) (2004).

${ }^{22}$ X. Feng and B. B. Laird, J. Chem. Phys. 124, 044707 (2006).

${ }^{23}$ J. Q. Broughton and G. H. Gilmer, J. Chem. Phys. 84, 5759 (1986).

${ }^{24}$ R. L. Davidchack and B. B. Laird, J. Chem. Phys. 118, 7651 (2003).

${ }^{25}$ J. Morris and X. Song, J. Chem. Phys. 119, 3920 (2003).

${ }^{26}$ M. Asta, J. J. Hoyt, and A. Karma, Phys. Rev. B 66, 100101(R) (2002).

${ }^{27}$ J. P. Hansen and I. R. McDonald, Theory of Simple Liquids, 3rd ed. (Academic, New York, 2006).

${ }^{28}$ B. B. Laird, J. Chem. Phys. 115, 2887 (2001).

${ }^{29}$ W. G. T. Kranendonk and D. Frenkel, J. Phys.: Condens. Matter 1, 7735 (1989).

${ }^{30}$ S. M. Foiles, Phys. Rev. B 32, 7685 (1985).

${ }^{31}$ W. G. T. Kranendonk and D. Frenkel, Mol. Phys. 72, 679 (1991).

${ }^{32}$ R. L. Davidchack and B. B. Laird, Phys. Rev. E 54, R5905 (1996).

${ }^{33}$ R. L. Davidchack and B. B. Laird, Mol. Phys. 97, 833 (1999).

${ }^{34}$ B. B. Laird and R. L. Davidchack, J. Phys. Chem. B 109, 17802 (2005).

${ }^{35}$ Y. Mu and X. Song, J. Chem. Phys. 124, 034712 (2006).

${ }^{36}$ R. Handel, R. L. Davidchack, J. Anwar, and A. Brukhno, Phys. Rev. Lett. 100, 036104 (2008).

${ }^{37}$ A. Karma, Phys. Rev. E 48, 3441 (1993).

${ }^{38}$ M. P. A. Fisher, D. S. Fisher, and J. D. Weeks, Phys. Rev. Lett. 48, 368 (1982).

${ }^{39}$ W. R. Fehlner and S. H. Vosko, Can. J. Phys. 54, 2159 (1976).

${ }^{40}$ R. L. Davidchack, J. R. Morris, and B. B. Laird, J. Chem. Phys. 125, 094710 (2006).

${ }^{41}$ J. J. Hoyt and M. Asta, Phys. Rev. B 65, 214106 (2002).

${ }^{42}$ D. C. Rapaport, The Art of Molecular Dynamics Simulation, 2nd ed. (Cambridge University Press, New York, 2004). 